\section{La necesidad de hacer reformas del sector de la salud orientadas hacia la equidad ${ }^{1}$}

\author{
Davidson R. Gwatkin ${ }^{2}$
}

\section{RESUMEN}

Se necesita una nueva ola de reformas del sector de la salud orientadas hacia la equidad que estén emprendidas con más pasión y empeño aun que las reformas efectuadas en los años noventa para aumentar la eficiencia. El objetivo de este trabajo es estimular más reflexión acerca de cómo conseguir este tipo de reformas mediante el planteamiento de tres argumentos:

- En primer lugar, hay un gran cuerpo de documentación que crece aceleradamente según el cual las intervenciones sanitarias que suelen constituir los componentes principales de los programas de salud para personas pobres benefician más a las personas más prósperas que a las más necesitadas. De ahí que se necesiten reformas más profundas de lo que se suele pensar si los sistemas de salud han de dar una ayuda eficaz a los pobres.

- En segundo lugar, el actual movimiento que busca eliminar la deuda de los países pobres está creando un clima general mucho más propicio a contemplar estos cambios profundos que el que había hace algún tiempo.

- En tercer lugar, hay una necesidad urgente de contar con una sólida base de ejemplos de intervenciones mejor encaminadas hacia las personas pobres, si el potencial que ofrece el actual clima ha de aprovecharse.

Palabras clave: equidad en salud, reforma del sector de la salud.

\footnotetext{
Esta es una traducción al español de Gwatkin DR, The need for equityoriented health sector reforms. Int J Epidemiol 2001;30(4):720-723.

2 Banco Mundial, Washington, D.C., Estados Unidos de América. Dirección postal: 1818 H Street, N.W., Washington, D.C., 20433, Estados Unidos de América.
}

\section{INTRODUCCIÓN}

Para muchas personas, la expresión "reforma del sistema de salud" evoca un conjunto particular de medidas que fueron adoptadas en muchos lugares en los años noventa y que se encaminaban a hacer frente a un problema particular: la mayor dependencia de las instituciones y procedimientos del sector privado para mejorar la eficiencia. En rigor, no obstante, la expresión "reforma del sistema de salud" es de carácter general y se aplica a una amplia variedad de políticas orientadas a lidiar con una serie de problemas de igual magnitud. De acuerdo con una definición estándar, por ejemplo, la reforma del sector de la salud se puede definir como "un cambio sostenido y intencionado que busca mejorar la eficiencia, equidad y eficacia del sector de la salud".

La adopción de una definición más amplia ofrece la oportunidad de superar el actual debate que se enfoca por entero en determinar si las reformas específicas que se han adoptado en el pasado han ayudado o perjudicado a los pobres, y de mirar desde una perspectiva más amplia las reformas que harán falta para beneficiar a los pobres en un futuro. Urge adoptar esta perspectiva, puesto que crece aceleradamente un cuantioso conjunto de pruebas de que hay grandes brechas entre las personas pobres y las más pudientes, no solo en el estado de salud y el uso de servicios de salud privados, sino también en el uso de servicios públicos, incluso los que gozan de una amplia promoción por suponerse que se dirigen a los pobres.

Esto apunta hacia la clara necesidad de una nueva ola de grandes reformas del sector de la salud, concebidas y aplicadas con más pasión y empeño aun que las reformas emprendidas en los años noventa para lograr una mayor eficiencia. El objetivo: aumentar la eficiencia y la eficacia con las que los sectores de la salud tienden la mano a los pobres y desfavorecidos, logrando así reducir las inequidades actuales en el uso de los servicios de salud y ayudando a subsanar las diferencias en el estado de la salud.

En las páginas siguientes se procurará suscitar mayor reflexión sobre la manera de conseguir tales reformas mediante el planteamiento de tres argumentos. El primero es que cualquier reforma de importancia implica cambios que son mucho más profundos de lo que suelen imaginar quienes defi- 
nen las políticas. El segundo es que el actual movimiento para eliminar la deuda de los países pobres está creando un clima mucho más abierto que el del pasado reciente a la realización de estos cambios profundos. El tercero es que los epidemiólogos e investigadores que estudian los sistemas de salud son quienes más pueden ayudar a los definidores de políticas que aspiran a lograr una mayor equidad a aprovechar el presente clima mediante el desarrollo de un conjunto de ejemplos de posibles intervenciones que lleguen a los pobres de un modo eficaz.

\section{LA NECESIDAD DE ADOPTAR REFORMAS MÁS PROFUNDAS}

La profundidad de las reformas que hacen falta para mejorar la equidad en el campo de la salud puede apreciarse cuando se examina la serie de iniciativas anteriores que se emprendieron en las postrimerías de los años setenta y principios de los ochenta para mejorar la equidad. En ese período predominaba una inquietud por los pobres del mundo, como se manifestaba en acontecimientos tan divulgados en los medios de comunicación como el movimiento de "salud para todos" que surgió como consecuencia de la Conferencia de Alma Ata celebrada en 1978 por la Organización Mundial de la Salud y el Fondo de las Naciones Unidas para la Infancia (UNICEF) (2) y de la "Revolución para la supervivencia infantil" que comenzó en 1982 (3). Entre otras cosas, tales sucesos dieron origen a enérgicas iniciativas de la OMS por extender el uso de la atención primaria de salud, y del UNICEF por promover el control del crecimiento corporal, la rehidratación oral, la lactancia materna y la vacunación. Este conjunto de procedimientos en inglés se ha llegado a conocer con el acrónimo de gobi (growth, oral rehydration, breastfeeding e immunization).

¿Cuán eficaces han sido estas iniciativas para llegar a la población pobre? No se puede llegar a ninguna conclusión que sea del todo satisfactoria. No obstante, algunos estudios recientes sobre las disparidades entre ricos y pobres en el uso de los servicios de salud ofrece fuertes indicios sobre la situación que existe actualmente con respecto a tres de las cinco medidas que hemos mencionado: la atención primaria de salud, la rehidratación oral y la vacunación.

La información sobre la atención primaria de salud procede de una serie de estudios del tipo llamado "beneficio-incidencia", que evalúa cómo se distribuyen en las distintas clases sociales los beneficios económicos que derivan de los diferentes gastos del gobierno. Se han realizado alrededor de dos docenas de estudios de beneficio-incidencia sobre el gasto del gobierno en la salud, la mayor parte bajo los auspicios del Banco Mundial, donde se originó la tradición. Los más informativos son una serie de estudios sobre siete países africanos, cuyas cifras se presentan en el cuadro 1 (4).

En los países que figuran en el cuadro para los cuales se cuenta con información, los ingresos y el consumo de servicios en el $20 \%$ más próspero de la población son de cinco a 25 veces más altos que entre el $20 \%$ más pobre (5). No obstante la frecuente invocación de la equidad como justificación para que el gobierno tome parte en la prestación de servicios de salud, los gastos del servicio de salud público que figuran en el cuadro suelen reforzar, en lugar de compensar, las inequidades observadas en los ingresos y el consumo.

Lo anterior significa que, en términos absolutos, los gastos del gobierno tienden a beneficiar a los africanos más ricos en lugar de los más pobres. En promedio, el $20 \%$ de la población de más altos recursos recibe más del doble del beneficio económico que recibe el $20 \%$ más pobre a partir de los gastos generales del gobierno en la salud (30\% frente a $12 \%$ del beneficio total). En el caso de la atención primaria, la razón de pobres a ricos en cuanto a beneficios es mucho más baja ( $23 \%$ frente a $15 \%)^{3}$ [sic.], lo cual indica, desde el punto de vista de la equidad, que la tendencia a favor de la atención primaria es decididamente un paso en la dirección correcta. Pero ya que el grupo más próspero devenga, de nuevo, la mitad [sic.] del beneficio económico que recibe el más pobre, incluso a partir de la atención primaria, difícil sería considerar la importancia de la medida como algo más que modesta. En otras partes del mundo en desarrollo la situación es menos sombría; pero no hay estudio efectuado en ningún país en el que el $20 \%$ más pobre de la población reciba ni siquiera el $25 \%$ del beneficio económico de los gastos del gobierno.

Sin lugar a dudas, son numerosas las limitaciones metodológicas que hay que tener presentes antes de llegar a ninguna apreciación definitiva de las cifras que representan la razón de beneficio a incidencia (5). Sin embargo, aun después de tomar estas cifras en cuenta, es difícil reconciliar el hallazgo de que la atención primaria confiere un mayor beneficio al $20 \%$ más rico que al $20 \%$ más pobre de la población de muchos países, con la visión que motivó a los autores de la Declaración de Alma Ata.

Últimamente han surgido datos sobre la rehidratación oral y la vacunación a partir de una serie de estudios basados en datos similares recolectados

\footnotetext{
3 Nota del traductor: En esta oración se habla de la razón de pobres a ricos (23:15), mientras que en la anterior se menciona la de ricos a pobres (30:12). El traductor sospecha que el autor ha cometido un error inadvertidamente y que en el segundo caso quiso decir ". . .la razón de ricos a pobres en cuanto a beneficios...". El error se repite en la oración siguiente.
} 
CUADRO 1. Porcentaje del total de los beneficios económicos derivados de los gastos estatales para la salud que favorecieron a los quintiles más pobre y más rico de la población

\begin{tabular}{|c|c|c|c|c|}
\hline \multirow[b]{2}{*}{ País } & \multicolumn{2}{|c|}{$\begin{array}{c}\text { Atención primaria } \\
\text { Porcentaje del beneficio que } \\
\text { favoreció al }\end{array}$} & \multicolumn{2}{|c|}{$\begin{array}{c}\text { Atención total } \\
\text { Porcentaje del beneficio que } \\
\text { favoreció al }\end{array}$} \\
\hline & Quintil más pobre & Quintil más rico & Quintil más pobre & Quintil más rico \\
\hline Côte d'Ivoire (1995) & 14 & 22 & 11 & 32 \\
\hline Ghana (1992) & 10 & 31 & 12 & 33 \\
\hline Guinea (1994) & 10 & 36 & 4 & 48 \\
\hline Kenya (rural - 1992) & 22 & 14 & 14 & 24 \\
\hline Madagascar (1993) & 10 & 29 & 12 & 30 \\
\hline Tanzanía (1992-93) & 18 & 21 & 17 & 29 \\
\hline África del Sur (1994) & 18 & 10 & 16 & 17 \\
\hline Media no ponderada & 15 & 23 & 12 & 30 \\
\hline
\end{tabular}

Fuente: Referencia 4

mediante encuestas domiciliarias en 44 países de África, Asia y América Latina (6). Estos estudios, que fueron comisionados por el Banco Mundial, arrojan los valores de alrededor de treinta indicadores sanitarios, nutricionales y poblacionales para cada quintil socioeconómico de la población. Los resultados de los estudios sobre rehidratación oral y vacunación se resumen en las figuras 1 y 2 .

De las dos intervenciones, la terapia de rehidratación oral cuenta con mejores antecedentes desde el punto de vista de la población pobre. En todas las grandes regiones del mundo, cerca de la mitad de los casos de diarrea infantil en el $20 \%$ más pobre de las familias han sido tratados con algún tipo de régimen oral a base de líquidos. Dada la dificultad para llegar a estas familias pobres, eso representa un logro importante para una tecnología que había existido apenas unos 20 años en el momento en que se recolectaron los datos. Pero aun en un caso como este, en que la tecnología se desarro-

FIGURA 1. Uso de la terapia de rehidratación oral (TRO) por pobres y ricos en países en desarrollo

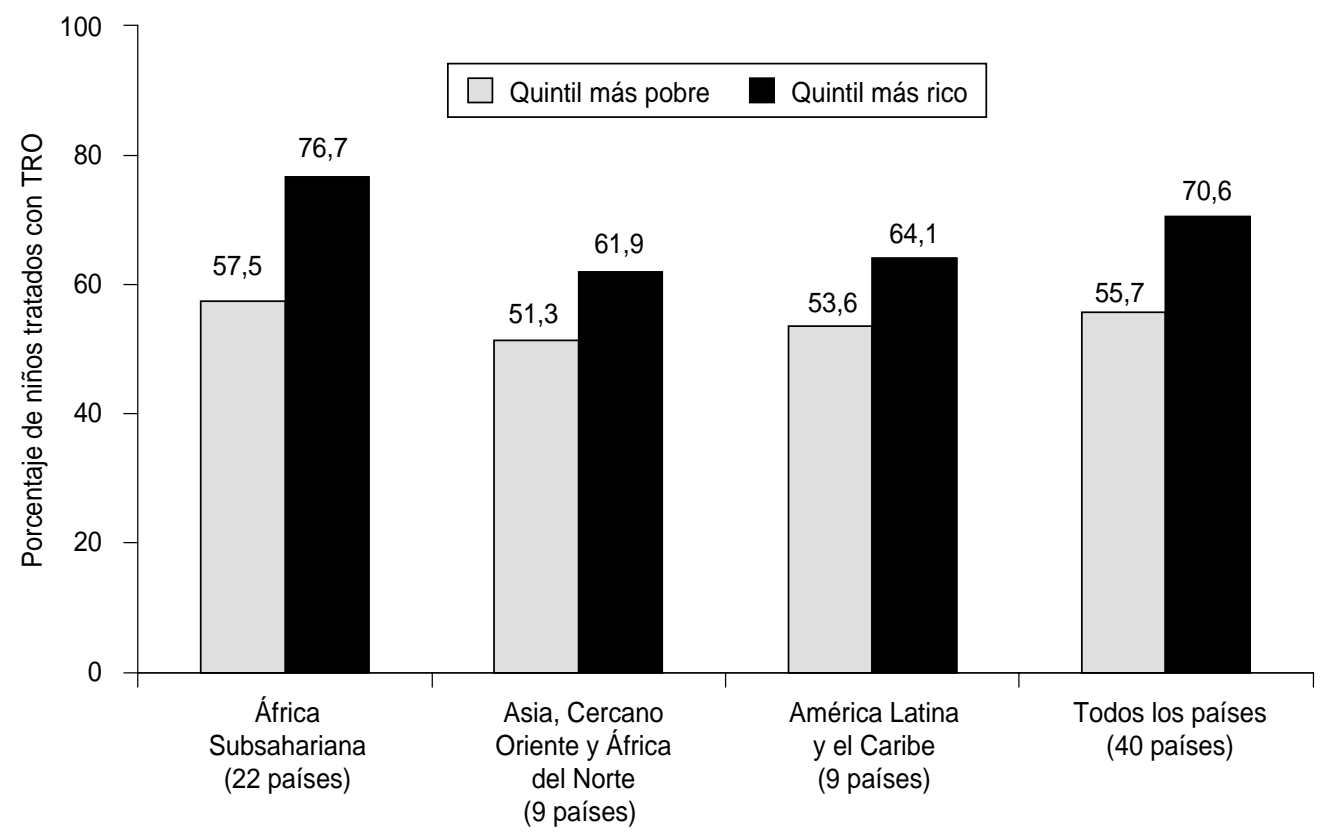

Porcentaje de niños menores de 3, 4 ó 5 años (según el país) con enfermedad diarreica que fueron tratados con sales de rehidratación oral o a quienes se les recomendó tomar líquidos o incrementar su ingestión, según posición socioeconómica, en países en desarrollo. 
FIGURA 2. Tasas de vacunación completa ${ }^{a}$ entre pobres y ricos en países en desarrollo

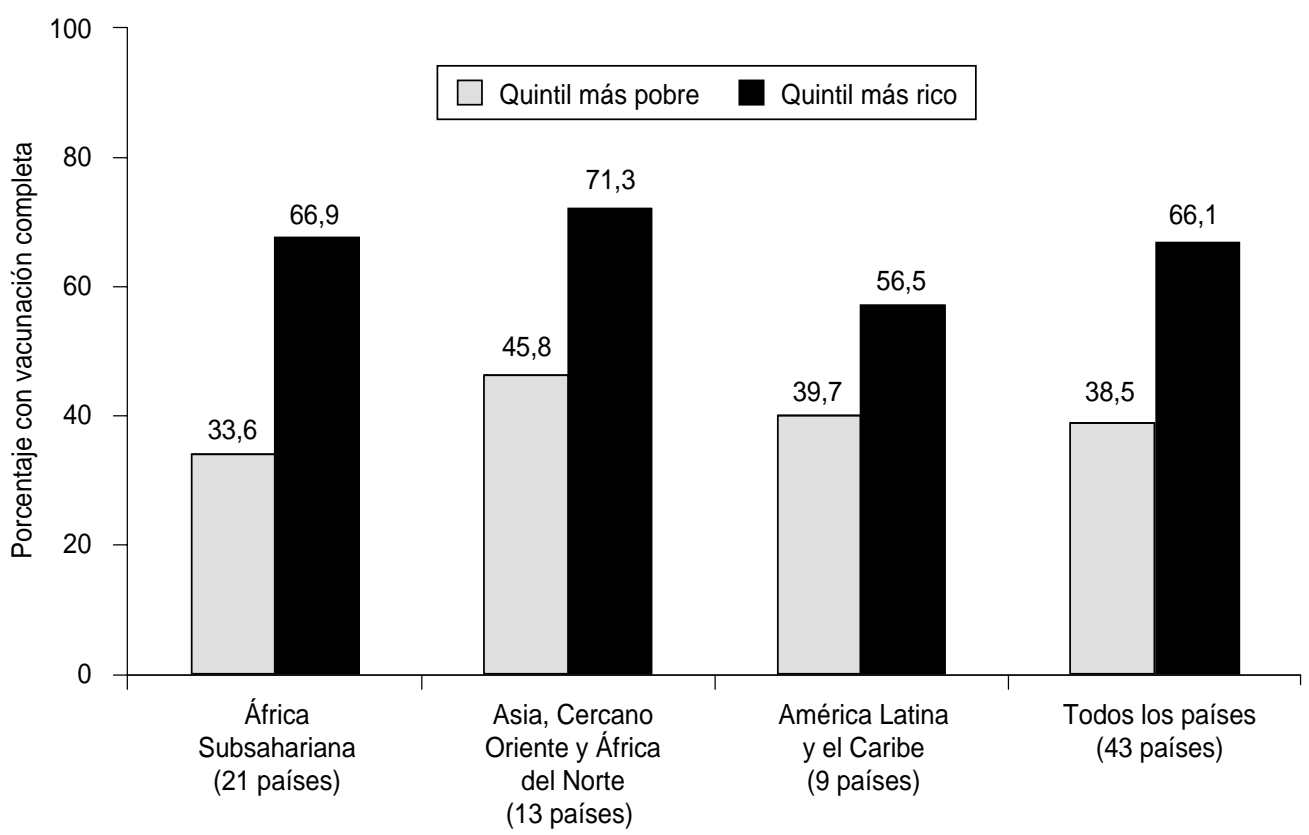

Fuente: Encuestas Demográficas y de Salud.

a La inmunización completa se refiere al porcentaje de niños vivos de 12-23 meses de edad que habían recibido las vacunas BCG y DPT más la vacuna antipoliomielítica oral y la vacuna antisarampionosa antes de la encuesta.

lló teniendo en mente a la población más pobre, la asimilación ha sido mayor entre las clases más adineradas, siendo las tasas de utilización alrededor de 10 a $20 \%$ más elevadas en el quintil más alto que en el más bajo. Y si bien es cierto que se ha logrado beneficiar a la mitad de los pobres, la otra mitad no ha devengado beneficio alguno; y no hay ningún indicio claro de que lo haga en el futuro próximo.

Si se examinan los datos de vacunación, la situación es aun menos alentadora. Aunque los datos que tenemos a la mano distan de ser perfectos, no dejan lugar a dudas de que las enfermedades prevenibles por vacunación son más frecuentes entre las personas pobres. No obstante, los programas de vacunación no están alcanzando a los pobres en igual medida que a los más ricos. En promedio, la cobertura de vacunación entre el $20 \%$ más pobre de la población es solo 35-40\%, apenas poco más de la mitad de la que tiene el $20 \%$ más próspero de la población de un país cualquiera, lo cual significa que los pobres se benefician mucho menos aun cuando se toman en cuenta las externalidades.

Si el vaso que se describe en los párrafos anteriores está lleno o vacío a la mitad depende, ineludiblemente, del punto de vista que se adopte. Desde el punto de vista de este observador, no pa- rece posible negar que los pobres se han beneficiado mucho de las innovaciones descritas y de las muchas otras de la misma índole que fueron introducidas en las postrimerías de los años setenta y principios de los ochenta. Sin embargo, es igualmente difícil negar que el beneficio ha sido relativamente modesto cuando se compara con la carga de morbilidad total que corresponde a los más pobres; $\mathrm{y}$, tal como se desprende de los datos aquí presentados, también es más pequeño que los beneficios aportados por las innovaciones a la población menos necesitada.

Lo anterior no equivale a decir que las innovaciones han sido un error. Si bien es cierto que no han logrado alcanzar a los pobres tan bien como lo anticipaban sus impulsores, también parece haberse demostrado que estaban más encaminadas a beneficiar a los pobres que los servicios de salud más viejos de los cuales se desvincularon. Si es así, las innovaciones pueden ser defendidas en tanto representan un primer paso en la dirección adecuada. Vistas retrospectivamente, sin embargo, resulta evidente que no han sido más que un primer paso. De por sí, no han sido suficientes, bajo ningún criterio, para superar las dificultades que plantea alcanzar a la población pobre de manera eficaz, dificultades 
estas cuya magnitud ha sido mucho mayor de la esperada, como demuestra la experiencia descrita anteriormente.

\section{El logro de reformas más profundas}

Si se da por cierto lo antedicho, se desprende que cualquier esfuerzo realmente eficaz para que la población pobre goce de los servicios que necesitan implicará una ruptura con el pasado aun más definitiva de lo que suele apreciarse. No se necesita solamente promover vigorosamente un halagador paquete de servicios como parte paralela de un sistema de salud como el que ahora existe, sino un cambio mucho más fundamental en la orientación del sistema en su totalidad: en otras palabras, una reforma profunda del sector de la salud.

Es más fácil abogar por tal reforma en la teoría que en la práctica. No obstante, en los últimos meses se han presenciado algunos acontecimientos que, si se aprovechan debidamente, podrían representar un primer paso en esa dirección: el afán por reducir la deuda externa de muchos países pobres.

Un componente fundamental de este afán ha sido la elaboración de estrategias para aliviar la pobreza por parte de un número cada vez mayor de gobiernos de países en desarrollo. Tales estrategias, incorporadas en documentos formales conocidos por "documentos para la estrategia de reducción de la pobreza", o DERP, tienen por meta a largo plazo proporcionar asesoramiento a los gobiernos en torno a sus estrategias generales en pro del desarrollo. La meta inmediata a corto plazo de los primeros gobiernos que participan en esta iniciativa es lograr que la deuda se reduzca.

Estos gobiernos son los de los cuarenta y un países con suficiente pobreza y endeudamiento para beneficiarse de la iniciativa para reducir parcialmente la deuda, propiciada por el Fondo Monetario y el Banco Mundial (conocida por Highly Indebted Poor Country initiative) (7). Según esta iniciativa, promovida activamente por un consorcio de agencias no gubernamentales, un país puede aspirar a reducir su deuda siempre que demuestre que el resultante aumento de los fondos públicos no adjudicados se destinará a actividades en beneficio de los pobres. La preparación de un DERP es la manera en que un gobierno da fe de lo anterior. Dicha preparación se inició a fines de 1999 y cobró impulso a lo largo del año 2000. Llegado marzo de 2001, cuatro países habían completado sus DERP definitivos; otros 31 habían completado DERP provisionales (8) y se elaboraban planes para extender la preparación de los DERP a todos los 78 países que cumplían los requisitos para recibir subsidios por conducto de la Asociación de Desarrollo Internacional del Banco Mundial (ADI).

Llegada la misma fecha (marzo de 2000), el Banco Mundial y el Fondo Monetario habían acordado reconocer cerca de $\$ 20$ mil millones (en valor actual neto) hacia el pago de sus deudas a 22 de los primeros 35 países que completaran DERP definitivos o provisionales. Los ministerios de salud a menudo han participado activamente en el proceso de elaboración de DERP, y cerca de la cuarta parte de los fondos entregados mediante el proceso de HIPC-DERP — cerca de \$5 mil millones- se habían destinado a programas de salud (9). La mayor parte de estos fondos destinados a la salud se habían adjudicado a la ampliación de programas similares a los que se hicieron populares a fines de los años setenta y comienzos de los ochenta y que se describieron en la sección anterior: atención primaria, vacunación, salud maternonfantil y demás.

A la luz de lo planteado en torno a las limitaciones que tienen los programas de este tipo para hacer llegar sus beneficios a los pobres, resulta evidente que se trata de medidas que, pese a su utilidad, distan de ser suficientes para constituir la reforma profunda que se necesita. Por consiguiente, el principal factor que explica el interés en estas medidas guarda menos relación con los beneficios que las mismas son capaces de aportar, que con el hecho de que ponen de manifiesto una ruptura largo deseada con la pasada década de inatención a las necesidades de los grupos desfavorecidos. Las medidas indican, por lo tanto, la posibilidad de una apertura, una oportunidad de luchar por conseguir reformas más fundamentales mediante el fortalecimiento, la profundización y la ampliación del proceso que el movimiento encaminado a reducir la deuda y preparar los DERP ha echado a andar. La pregunta es si las medidas se tomarán de esa manera, con lo que servirán para iniciar una serie de cambios profundos, o si no serán más que un esfuerzo por implantar las iniciativas definidas actualmente, produciendo así un beneficio a lo sumo modesto.

\section{LA FUNCIÓN DE LOS EJEMPLOS}

Dado el papel central que desempeña la política interna en cualquier reforma sanitaria a fondo, los funcionarios públicos y los que definen las políticas de los países implicados tendrán, por fuerza, que ser los principales responsables de contestar la pregunta que acabamos de plantear. Pero muchas otras entidades también desempeñarán un papel secundario. Uno de ellos, para el cual los sistemas epidemiológicos y sanitarios están especialmente bien preparados, es el desarrollo de una base de 
datos fidedigna con ejemplos de las distintas intervenciones que se podrían aplicar.

Este es un elemento cuya ausencia se destaca en la labor recién emprendida por epidemiólogos y otros investigadores que manifiestan una inquietud creciente por la salud de los más pobres y la equidad en ese aspecto. Hasta la fecha, la labor ha producido aumentos importantes en los conocimientos sobre la magnitud y naturaleza de las inequidades en el campo de la salud y ha dado origen a valiosos marcos conceptuales para abordar estas cuestiones. Pero aún no ha llegado al meollo del asunto: la identificación de medidas que puedan subsanar eficazmente las inequidades que han sido descubiertas.

La identificación de tales medidas representa la próxima frontera para las investigaciones epidemiológicas y sanitarias relacionadas con la equidad y la salud de los pobres. Como dijeran quienes participaron en una consulta reciente organizada por la Fundación Rockefeller en colaboración con el Banco Mundial, la principal necesidad en el ámbito de la investigación en lo que respecta a la equidad es "desviar el actual énfasis estático sobre la medición y el análisis de las inequidades en materia de salud hacia una identificación y evaluación dinámicas de las politicas que pueden conseguir una mayor equidad de manera eficaz" (10). (El énfasis es propio del original.)

Es especialmente urgente hacer evaluaciones empíricas sólidas de la eficacia con que las actuales iniciativas sanitarias y de desarrollo benefician a los pobres y lograr un mayor conocimiento de lo que hay que hacer para mejorar su utilidad, así como experimentar sobre el terreno con enfoques nuevos y promisorios. $Y$ estas cosas hacen falta de inmediato si se ha de aprovechar el actual ímpetu que tienen las medidas para aliviar la pobreza, tal como indica el movimiento actual encaminado a este fin. Hasta que no se produzcan enfoques de este tipo, los gobiernos de países en desarrollo que deseen reorientar sus programas de salud hacia los pobres en mayor medida no tendrán otra opción que la de seguir aferrados a los fallidos antecedentes que nos brinda el pasado como fuente de inspiración para idear programas. Y como resultado, los pobres seguirán siendo servidos pobremente.

\section{REFERENCIAS}

1. Berman PA. Health sector reform: making health development sustainable. En: Berman PA, ed. Health sector reform in developing countries: making health development sustainable. Boston: Harvard School of Public Health 1995. P. 15.

2. World Health Organization. Primary health care. Report of the International Conference on Primary Health Care, Alma Ata, USSR, 6-12 September 1978. Geneva: WHO; 1978.

3. Grant JP. The state of the world's children, 1982-83. New York y otras ciudades: Oxford University Press; 1982.
4. Castro-Leal F, Dayton J, Mehra K. Public spending on health care in Africa: do the poor benefit? Bull World Health Organ 2000;78(1):66-74.

5. World Bank. World development report 2000/2001: attacking poverty. Washington, D.C.: WB; 2000

6. Gwatkin DR, Rutstein S, Johnson K, Pande R, Wagstaff A. Socioeconomic differences in health, nutrition, and population. Washington, D.C.: Health, Nutrition, and Population Department of the World Bank; 2000. También se encuentra en el siguiente sitio: www.worldbank. org/poverty/health/data/index.htm
7. Se encuentra en www.worldbank.org/ hipc/about/hipcbr.htm

8. Se encuentra en www.worldbank.org/ prsp/

9. HIPC Unit, World Bank, Financial impact of the HIPC Initiative: first 22 country cases, March 1, 2001. www.worldbank. org/hipc/hipc-review/hipc-review. html

10. Rockefeller Foundation. Current activities and future directions in health equity: a consultation with NGOs, researchers, foundations, and bilaterals, June 23-24, 1999. (Documento mimeografiado). 